\title{
Effects of electric charge on osmotic flow across periodically arranged circular cylinders
}

\author{
MASAKO SUGIHARA-SEKI†, TAKESHI AKINAGA, \\ AND TOMOAKI ITANO \\ Department of Pure and Applied Physics, Kansai University, 3-3-35 Yamate-cho, Suita, \\ Osaka 564-8680, Japan
}

(Received 19 October 2009; revised 30 June 2010; accepted 8 July 2010; first published online 27 September 2010)

An electrostatic model is developed for osmotic flow across a layer consisting of identical circular cylinders with a fixed surface charge, aligned parallel to each other so as to form an ordered hexagonal arrangement. The expression of the osmotic reflection coefficient is derived for spherical solutes with a fixed surface charge suspended in an electrolyte, based on low-Reynolds-number hydrodynamics and a continuum, point-charge description of the electric double layers. The repulsive electrostatic interaction between the surface charges with the same sign on the solute and the cylinders is shown to increase the exclusion region of solute from the cylinder surface, which enhances the osmotic flow. Applying the present model to the study of osmotic flow across the endothelial surface glycocalyx of capillary walls has revealed that this electrostatic model could account well for the reflection coefficients measured for charged macromolecules, such as albumin, in the physiological range of charge density and ion concentration.

Key words: biomedical flows, biological fluid dynamics, low-Reynolds-number flows

\section{Introduction}

Osmotic flow is generated between solutions of different concentrations that are separated by a porous membrane. When only solvent can enter the pores and the solutes are impermeable, a net volume flux of the solvent, referred to as the osmotic flow, is realized. If the solutes are only partially excluded from the membrane, then an osmotic reflection coefficient, $\sigma_{v}$, must be introduced so as to express the solvent flux across the leaky membrane. In this case, the thermodynamic principles yield the following expression for the solvent flux $J_{v}$ per unit cross-sectional area when a hydrostatic pressure difference $\left(\Delta p_{\infty}\right)$ and osmotic pressure difference $\left(\Delta \pi_{\infty}\right)$ are applied across the membrane

$$
J_{v}=L_{p}\left(\Delta p_{\infty}-\sigma_{v} \Delta \pi_{\infty}\right),
$$

where $L_{p}$ is the hydraulic permeability and $\infty$ denotes the bulk solution conditions on both sides of the membrane. The accompanying expression for the solute flux $J_{s}$ is

$$
J_{s}=\omega \Delta \pi_{\infty}+\left(1-\sigma_{s}\right) c^{*} J_{v},
$$

$\dagger$ Email address for correspondence: sekim@ipcku.kansai-u.ac.jp 
where $\omega$ is the diffusive permeability, $\sigma_{s}$ is the filtration reflection coefficient and $c^{*}$ is the average concentration of the solute (Kedem-Katchalsky equation; Kedem \& Katchalsky 1958; Curry 1984; Truskey, Yuan \& Katz 2004). The first term on the right-hand side of (1.1) represents the fluid flux that is proportional to the pressure difference and the second term represents the osmotic flow. It is to be noted that for semipermeable membranes, $\sigma_{v}=1$ and $\sigma_{v}$ tends to zero as the solute size approaches zero. If Onsager's reciprocity postulate is assumed, it can be shown that $\sigma_{s}$ and $\sigma_{v}$ are equal (Levitt 1975).

In previous studies (Sugihara-Seki 2006; Sugihara-Seki, Akinaga \& Itano 2008), we investigated the transport of solute and solvent across the fibre-matrix layer of proteoglycans and glycoproteins that is present at the surface of vascular endothelial cells (Vink \& Duling 1996, 2000; Pries, Secomb \& Gaehtgens 2000; Weinbaum et al. 2003; Reitsma et al. 2007; Weinbaum, Tarbell \& Damiano 2007). This layer is referred to as the endothelial surface glycocalyx layer (EGL). By using a simplified model of the EGL, we analysed the motion of solute and solvent to estimate the filtration reflection coefficient, $\sigma_{s}$, as well as the diffusive permeability, $\omega$, for the EGL based on (1.2). In the simplified model, core proteins in the EGL were assumed to have a circular cylindrical shape and to be aligned in parallel to form a hexagonal arrangement, based on recent detailed structural analyses of the EGL (Squire et al. 2001; Weinbaum et al. 2003); see figure 1. We computed the drag force and the torque exerted on a spherical solute suspended in a fluid flowing between these periodically aligned cylinders, and the obtained values were used to estimate $\omega$ and $\sigma_{s}$ from thermodynamic and mechanical equilibrium relations. The predicted values of $\omega$ and $\sigma_{s}$ exhibited reasonable agreement with experimental observations (Sugihara-Seki 2006).

Zhang, Curry \& Weinbaum (2006) studied osmotic flow through the EGL model using a method developed by Anderson \& Malone (1974) for osmotic flow in porous membranes. From considerations of classical transport and thermodynamics, Anderson \& Malone (1974) showed that the presence of an exclusion region of solute near the pore walls produces a radial discontinuity in hydrostatic pressure and solute concentration, which generates the driving force for the osmotic flow. Zhang et al. (2006) applied this formulation to the osmotic flow across the EGL model consisting of hexagonally arranged cylinders. Instead of a rigorous treatment of the hexagonal geometry of the cylinders, they adopted an approximation in which the geometry is replaced by an equivalent fluid annulus around each cylinder and estimated the osmotic reflection coefficient, $\sigma_{v}$, for the EGL based on (1.1). Despite the approximate geometry adopted by Zhang et al. (2006), the values obtained for $\sigma_{v}$ were comparable to the results for $\sigma_{s}$ obtained by Sugihara-Seki (2006), which is consistent with Onsager's reciprocal theory.

Since biological molecules are often electrically charged, significant contributions of electric charge to material transport have been reported for capillaries in various tissues (Curry 1984). For example, Adamson, Huxley \& Curry (1988) reported that for ribonuclease and $\alpha$-lactalbumin, which are solutes of similar size, the permeability of ribonuclease with positive charge was twice that of $\alpha$ lactalbumin with negative charge in frog mesenteric capillaries. Vink \& Duling (2000) demonstrated that, for anionic molecules, in addition to size, the electric charge has a profound effect on the penetration rate into the EGL. Using cultured endothelial cells, Ueda et al. (2004) showed that the albumin uptake for the endothelial cells with a neutralized (no charge) EGL was almost twice that of cells with charged EGL. 
Previously, a simple Donnan-type model was proposed to describe the charge effect on microvessel permeability (Adamson et al. 1988; Curry, Rutledge \& Lenz 1989; Huxley \& Curry 1991; Huxley et al. 1993; Fu, Chen \& Chen 2003). From in vivo measurements of solute-permeability coefficients, Adamson et al. (1988) estimated the charge density of frog mesenteric capillary to be approximately $-10 \mathrm{mEq} 1^{-1}$ by considering the electric partition. Fu et al. (2003) developed an electrodiffusion model for the EGL and examined the diffusive permeability of positively or negatively charged macromolecules as a function of charge density in the EGL. Stace \& Damiano (2001) and Damiano \& Stace (2002) considered the electrochemical dynamics of anionic molecular transport within capillaries by modelling the EGL as a continuously distributed anionic matrix containing fixed-bound negative charges through which a solution of anionic molecular tracers in blood can diffuse.

In a previous study (Akinaga, Sugihara-Seki \& Itano 2008), we examined the charge effect on the osmotic flow for membranes with circular cylindrical pores by extending the formulation of osmotic flow developed by Anderson \& Malone (1974). We considered the case in which the surfaces of the solute and the pore walls are negatively charged, and the solvent is an electrolyte containing small ions. By using a mean-field theory for the electric field around a solute in a cylindrical pore, we evaluated the osmotic reflection coefficient, $\sigma_{v}$, under electrostatic effects. The repulsive electrostatic interaction between the surface charges was shown to increase the exclusion region from the pore wall and, consequently, to significantly enhance the osmotic flow.

In this paper, we apply our electrostatic model for the osmotic flow to the EGL and examine the effect of surface charges on the osmotic reflection coefficient, $\sigma_{v}$. The EGL model in our previous study (Sugihara-Seki 2006) is modified such that the cylinders and solute have fixed surface charges and the solvent is an electrolyte. We restrict the analyses to the case of like charges, i.e. repulsive interaction between surface charges. In this case, the solute is more likely to be excluded from the cylinder surface due to the repulsive interaction, which may largely affect the values of the reflection coefficient.

The ions in the electrolyte are assumed to be sufficiently small, so that we adopt a continuum, point-charge description of the electric double layers. The electrostatic potential around a solute in the electrolyte is determined by the Debye-Hückel $(\mathrm{DH})$ equation, which is a linearized form of the Poisson-Boltzmann (PB) equation. These equations can be derived from the mean-field approximation that ignores fluctuations in the electrostatic potential, and thus neglects correlations between ions in the electrolyte. These equations have been subjected to extensive formal and computational tests, and the validity and limitation of their applicability in various systems have been investigated (Reiner \& Radke 1991). Beyond this limitation, charge-correlation effects can lead to charge inversion and flocculation in aqueous solutions containing multivalent counterions (Likos 2001; Levin 2002). In particular, anomalous behaviours of biopolymers, such as cytoskeleton organization and the compaction of generic materials in biological systems, may be closely related to these effects (Levin 2002). However, in this study, we do not include charge correlations between ions. Polarization effects, e.g. charge-induced dipole interactions, are also neglected. Furthermore, we include neither electrostatic interactions between solutes nor their fluid dynamic interactions, considering the dilute limit of zero solute concentration.

In $\S 2$, we develop an electrostatic model for the osmotic flow across the EGL with an electric charge and outline the procedures used to obtain the osmotic reflection 


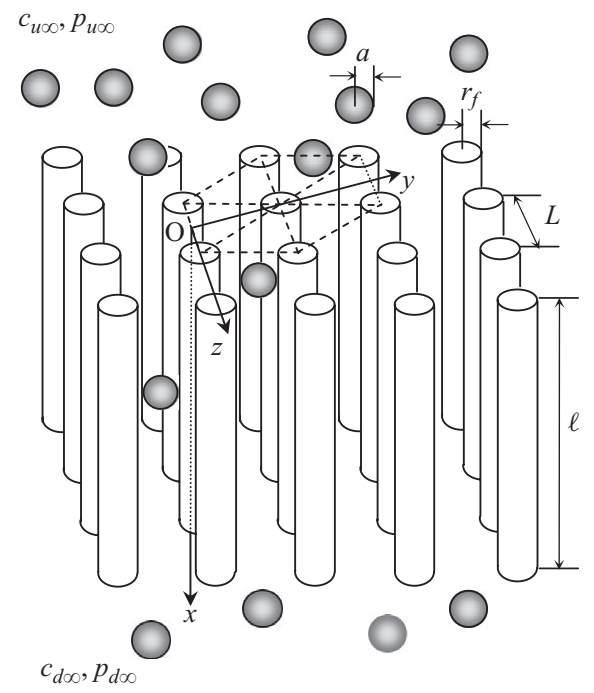

FIGURE 1. Sketch of the EGL model consisting of hexagonally arranged circular cylinders of radius $r_{f}$. The distance between adjacent cylinders is $L$, and the length of the cylinders is $\ell$. Spherical solutes of radius $a$ are suspended in an electrolyte, which contains small ions. The surfaces of the solute and the cylinders are electrically charged with densities $q_{s}$ and $q_{c}$, respectively. On both sides of the EGL model, there are bulk solutions with solute concentration $c_{u \infty}, c_{d \infty}$ and hydrostatic pressure $p_{u \infty}, p_{d \infty}$, respectively.

coefficient. Sections 3 and 4 present the results and discussion, respectively, regarding the dependence of the osmotic reflection coefficient on the surface charge as well as the ion concentration of the electrolyte.

\section{Formulation and methods}

\subsection{Endothelial surface glycocalyx layer model}

We consider an osmotic flow across a layer consisting of circular cylinders of radius, $r_{f}$, that are aligned parallel to each other to form a hexagonal arrangement of length, $L$, as shown in figure 1. The cross-section of the geometry is shown in figure 2 . The width of the layer or the axial length of the cylinders, $\ell$, is assumed to be large enough compared to $L$, so that the end effects on the fluid motion and the electric field can be neglected. On both sides of the layer, there are two solutions having different solute concentrations $\Delta c_{\infty}=c_{u \infty}-c_{d \infty}$ and hydrostatic pressures $\Delta p_{\infty}=p_{u \infty}-p_{d \infty}$. The difference in solute concentrations is equivalent to the difference in osmotic pressure, $\Delta \pi_{\infty}=\pi_{u \infty}-\pi_{d \infty}$, where $\pi_{u \infty}=c_{u \infty} R T$ and $\pi_{d \infty}=c_{d \infty} R T$. Here, $R$ is the gas constant and $T$ is the absolute temperature of the solution that is assumed to be constant.

The solutes are identical rigid spheres of radius $a$ (see figure 1). The surfaces of the cylinders and the solutes are assumed to have uniformly distributed electric charges with surface densities of $q_{c}$ and $q_{s}$, respectively. The solutes are suspended in an electrolyte. The sizes of the ions in the electrolyte are assumed to be small when compared to $r_{f}$ and $a$, so that the ions are regarded as point charges and the electrolyte is regarded as a continuum. We assume that the suspending fluid is an incompressible Newtonian fluid with viscosity $\mu$. The solution is assumed to be sufficiently dilute, so that the interaction between the solutes can be neglected. We 


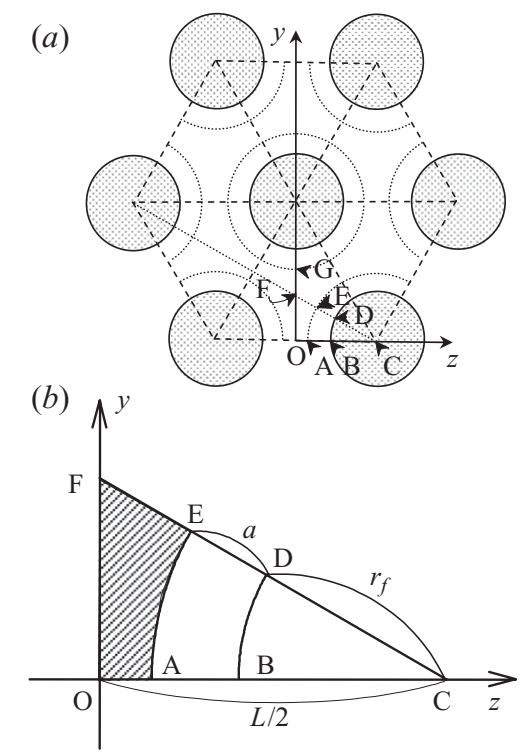

FIGURE 2. Cross-section of the hexagonal arrangement of circular cylinders. (a) Unit of the hexagonal geometry. (b) Typical equivalent triangular region.

also assume that there is no difference in ion concentrations on both sides of the EGL.

\subsection{Expression of the osmotic reflection coefficient $\sigma_{v}$}

We take the $x$-axis to be along the centreline between two adjacent cylinders, with the origin at the entrance to the EGL. The $y$ - and $z$-axes are taken to be in the plane perpendicular to the cylinder axes, as shown in figures 1 and 2 . We denote the fluid velocity as $\boldsymbol{u}=(u, v, w)$, the pressure as $p$, the concentration of the solutes as $c$ (or the osmotic pressure $\pi=c R T$ ) and the potential for the solute as $\phi$. The potential $\phi(x, y, z)$ is defined relative to the reservoirs on both sides of the EGL, where the value of the potential is zero. The electric charge density and the electric double layer potential in the electrolyte are denoted as $\rho_{e}$ and $\psi$, respectively.

For the bulk flow of solution containing solutes and small ions, the Stokes equation is expressed as follows:

$$
-\nabla p+\mu \nabla^{2} \boldsymbol{u}-c \nabla \phi-\rho_{e} \nabla \psi=\mathbf{0},
$$

which differs from the expression in Anderson \& Malone (1974) by including the electric body force. Since the cross-section is uniform and the length of the cylinders is much larger than the typical length in the cross-section, $L$, we assume that $|u| \gg$ $|v|,|w|$, and $\boldsymbol{u}=\boldsymbol{u}(y, z)$ and $\phi=\phi(y, z)$. Similarly, since $\rho_{e}$ and $\psi$ represent bulk quantities, we assume that $\rho_{e}=\rho_{e}(y, z)$ and $\psi=\psi(y, z)$.

Using the half-distance between adjacent cylinders, $L / 2$, we non-dimensionalize the length, such that $x^{*}=x /(L / 2), y^{*}=y /(L / 2)$ and $z^{*}=z /(L / 2)$. Similarly, we define $a^{*}=a /(L / 2), r_{f}^{*}=r_{f} /(L / 2)$ and $\ell^{*}=\ell /(L / 2)$. We also introduce dimensionless quantities $\boldsymbol{u}^{*}, p^{*}$ and $\pi^{*}$, using a representative axial velocity $V: \boldsymbol{u}^{*}=\boldsymbol{u} / V$, $p^{*}=p(L / 2 \mu V)$ and $\pi^{*}=\pi(L / 2 \mu V)$. The solute potential and the electrostatic potential are non-dimensionalized as $\phi^{*}=\phi / R T$ and $\psi^{*}=F \psi / R T$, respectively, where $F$ denotes the Faraday constant. 
Under the above assumptions, we obtain the simplified equation for the axial velocity in a dimensionless form from (2.1):

$$
-\frac{\partial p^{*}}{\partial x^{*}}+\Delta_{2} u^{*}=0, \quad \text { where } \quad \Delta_{2}=\frac{\partial^{2}}{\partial y^{* 2}}+\frac{\partial^{2}}{\partial z^{* 2}} .
$$

For the velocity components in the $y^{*}$ - and $z^{*}$-directions, (2.1) yields the following approximate equations:

$$
\frac{\partial p^{*}}{\partial y^{*}}+\pi^{*} \frac{\partial \phi^{*}}{\partial y^{*}}+\rho_{e}^{*} \frac{\partial \psi^{*}}{\partial y^{*}}=0, \quad \frac{\partial p^{*}}{\partial z^{*}}+\pi^{*} \frac{\partial \phi^{*}}{\partial z^{*}}+\rho_{e}^{*} \frac{\partial \psi^{*}}{\partial z^{*}}=0
$$

where the electric charge $\rho_{e}$ is non-dimensionalized appropriately. It is to be noted that (2.3) represents the mechanical equilibrium in the cross-section, indicating the generation of pressure gradients in the $y^{*}$ - and $z^{*}$-directions, respectively. If we further assume thermodynamic equilibrium, we obtain the Boltzmann distribution of the solutes as follows:

$$
\pi^{*}\left(x^{*}, y^{*}, z^{*}\right)=\pi_{0}^{*}\left(x^{*}\right) \exp \left[-\left(\phi^{*}\left(y^{*}, z^{*}\right)-\phi_{0}^{*}\right)\right],
$$

where $\phi_{0}^{*}=\phi^{*}(1 / \sqrt{3}, 0)$ is the potential at reference point $\mathrm{F}$ (see figure 2 ). The subscript 0 indicates values at point $\mathrm{F}$. Note that point $\mathrm{F}$ is located equidistantly from three adjacent cylinders.

Substituting (2.4) into (2.3) and integrating the resulting equation yields

$$
p^{*}\left(x^{*}, y^{*}, z^{*}\right)=p_{0}^{*}\left(x^{*}\right)-\pi_{0}^{*}\left(x^{*}\right)\left\{1-\exp \left[-\left(\phi^{*}\left(y^{*}, z^{*}\right)-\phi_{0}^{*}\right)\right]\right\}+g^{*}\left(y^{*}, z^{*}\right),
$$

where $g^{*}\left(y^{*}, z^{*}\right)$ represents the integrated form of the last term on the left-hand side of (2.3). For a univalent-univalent electrolyte, using the PB equation, we obtain $g^{*}\left(y^{*}, z^{*}\right)=\beta\left(\cosh \psi^{*}\left(y^{*}, z^{*}\right)-\cosh \psi_{0}^{*}\right)$, where $\beta=C_{\infty} L R T /(\mu V)$ and $C_{\infty}$ represents the ion concentration in the reservoirs on both sides of the EGL. As shown below, the term $g^{*}$ does not contribute to the osmotic flow, and therefore is not considered hereinafter.

Equation (2.5) illustrates the coupling between osmotic and hydrostatic pressures, which generates the driving force for the bulk flow. Then, from (2.2), we obtain

$$
\Delta_{2} u^{*}=p_{0}^{* \prime}-\pi_{0}^{* \prime}+\pi_{0}^{* \prime} \exp \left[-\left(\phi^{*}\left(y^{*}, z^{*}\right)-\phi_{0}^{*}\right)\right]
$$

where the prime represents $\mathrm{d} / \mathrm{d} x^{*}$. Since this equation is linear in $u^{*}$, its solution can be expressed in the form

$$
u^{*}\left(y^{*}, z^{*}\right)=-p_{0}^{* \prime} u_{1}^{*}+\pi_{0}^{* \prime}\left(u_{1}^{*}-u_{2}^{*}\right)
$$

where $u_{1}^{*}\left(y^{*}, z^{*}\right)$ and $u_{2}^{*}\left(y^{*}, z^{*}\right)$ are the solutions of the following equations:

$$
\Delta_{2} u_{1}^{*}=-1, \quad \Delta_{2} u_{2}^{*}=-\exp \left[-\left(\phi^{*}\left(y^{*}, z^{*}\right)-\phi_{0}^{*}\right)\right] .
$$

We apply the no-slip condition on the surface of the cylinders for $u_{1}^{*}$ and $u_{2}^{*}$. The second term on the right-hand side of (2.7) represents the osmotic flow that is proportional to the osmotic pressure gradient $\pi_{0}^{* \prime}$. From $(2.8 a)$ and $(2.8 b), u_{1}^{*}$ is the flow due to a constant pressure gradient along the cylinder axis, while $u_{2}^{*}$ is caused by the solute-wall interaction $\phi^{*}\left(y^{*}, z^{*}\right)$, which generates variation in the driving force in the cross-section. With regard to the osmotic flow, $u_{1}^{*}-u_{2}^{*}$, note that the first term, $u_{1}^{*}$, is the flow in the direction from the dilute to the concentrated side of EGL, whereas the second term, $u_{2}^{*}$, is the flow in the opposite direction.

For the range of $\left(y^{*}, z^{*}\right)$ in (2.8), we confine ourselves to region OBDF shown in figure 2(a), because the hexagonal cross-section can be divided into equal triangular 
regions, such as OCF (see figure $2 b$ ). The area of region OBDF is $A^{\prime}=1 / 2 \sqrt{3}-$ $\pi r_{f}^{* 2} / 12$. By integrating (2.7) over region OBDF, we obtain the flux, $U^{*}$, of the solution as

$$
U^{*}=-p_{0}^{* \prime} U_{1}^{*}+\pi_{0}^{* \prime}\left(U_{1}^{*}-U_{2}^{*}\right)
$$

where

$$
U_{1}^{*}=\frac{1}{A^{\prime}} \int_{A^{\prime}} u_{1}^{*} \mathrm{~d} S, \quad U_{2}^{*}=\frac{1}{A^{\prime}} \int_{A^{\prime}} u_{2}^{*} \mathrm{~d} S
$$

Note that $U^{*}$ is the mean velocity averaged over the cross-sectional area $A^{\prime}$, and, according to the continuity condition, its value is constant independent of $x^{*}$. Therefore, by neglecting the end effect, one can integrate (2.9) along the $x^{*}$-axis from the entrance $\left(x^{*}=0\right)$ to the exit $\left(x^{*}=\ell^{*}\right)$ to obtain

$$
U^{*}=\left(\frac{U_{1}^{*}}{\ell^{*}}\right)\left\{\left(p_{0}^{*}(0)-p_{0}^{*}\left(\ell^{*}\right)\right)-\left(\pi_{0}^{*}(0)-\pi_{0}^{*}\left(\ell^{*}\right)\right)\left(1-\frac{U_{2}^{*}}{U_{1}^{*}}\right)\right\} .
$$

The Boltzmann and Gibbs-Duhem relations are applied at the cylinder ends to obtain

$$
\begin{aligned}
& p_{0}^{*}(0)-p_{0}^{*}\left(\ell^{*}\right)=\Delta p_{\infty}^{*}-\Delta \pi_{\infty}^{*}\left(1-\mathrm{e}^{-\phi_{0}^{*}}\right), \\
& \pi_{0}^{*}(0)-\pi_{0}^{*}\left(\ell^{*}\right)=\Delta \pi_{\infty}^{*} \mathrm{e}^{-\phi_{0}^{*}},
\end{aligned}
$$

where $\Delta p_{\infty}^{*}$ and $\Delta \pi_{\infty}^{*}$ are dimensionless pressure difference and osmotic pressure difference, respectively, in the bulk fluid phases across the EGL (see figure 1). Equation (2.11) is finally reduced to the dimensionless form of (1.1),

$$
J_{v}^{*}=\alpha U^{*}=\alpha\left(\frac{U_{1}^{*}}{\ell^{*}}\right)\left\{\Delta p_{\infty}^{*}-\Delta \pi_{\infty}^{*}\left[1-\left(\frac{U_{2}^{*}}{U_{1}^{*}}\right) \mathrm{e}^{-\phi_{0}^{*}}\right]\right\},
$$

where $\alpha$ is the void fraction of the EGL: $\alpha=A^{\prime} / A$. Here, $A$ is the area of triangular region OCF in figure 2 , which is given in dimensionless form by $A=1 / 2 \sqrt{3}$. The corresponding dimensional form is

$$
J_{v}=L_{p}\left(\Delta p_{\infty}-\sigma_{v} \Delta \pi_{\infty}\right)
$$

and

$$
L_{p}=\alpha \frac{U_{1}^{*}}{\mu \ell}\left(\frac{L}{2}\right)^{2}, \quad \sigma_{v}=1-\frac{U_{2}^{*}}{U_{1}^{*}} \exp \left(-\phi_{0}^{*}\right)
$$

where $U_{1}^{*}$ and $U_{2}^{*}$ are obtained from (2.8) and (2.10). If the cross-section of the geometry is circular, rather than hexagonal, $(2.8 a)$ indicates that velocity $u_{1}^{*}$ coincides with a Poiseuille flow driven by a unit pressure gradient. In this case, we obtain $U_{1}^{*}=1 / 8$ and $L_{p}=\alpha(D / 2)^{2} / 8 \mu \ell$, where $D$ represents the diameter of the circular cross-section. For the hexagonal cross-section, an analytical solution for $(2.8 a)$ was obtained by Sparrow \& Loeffler $\operatorname{Jr}(1959)$, as shown in figure 5(a).

Equation $(2.8 b)$ does not have analytical solutions. In general, we need to solve $(2.8 b)$ numerically, for given potentials $\phi^{*}$. If the electric charge is absent, the steric condition yields $\phi^{*}\left(y^{*}, z^{*}\right)=0$ in region OAEF and $\phi^{*}\left(y^{*}, z^{*}\right)=\infty$ in region ABDE in figure 2. In this case, $\sigma_{v}$ is a function of only geometric parameters. In the presence of electrostatic effects, $\sigma_{v}$ also depends on the surface charges and the ion concentration in the electrolyte. We must first evaluate the solute potential energy $\phi^{*}$ under the electrostatic interaction. 


\subsection{Interaction energy}

Since we consider a dilute solution and neglect the mutual interaction between solutes, we treat a single solute present in the hexagonal cross-section, the centre of which is located at $\left(y^{*}, z^{*}\right)=\left(c_{y}^{*}, c_{z}^{*}\right)$. From symmetry considerations, the range of $\left(c_{y}^{*}, c_{z}^{*}\right)$ can be confined to the hatched region of OAEF in figure $2(b)$. In $\S 2.3$, we show how to evaluate the solute potential energy $\phi^{*}$, from the electrostatic interaction energy between the solute surface charge (density $q_{s}$ ) and the cylinder surface charge (density $q_{c}$ ).

According to the mean-field theory, the electrostatic potential $\Psi$ around a solute in the electrolyte is determined by Poisson's equation, which is expressed in a dimensional form as follows:

$$
\nabla^{2} \Psi=-\frac{F}{\varepsilon} \sum_{i} z_{i} C_{i},
$$

where $C_{i}$ represents the concentration of electrolyte species $i, z_{i}$ is its valence and $\varepsilon$ is the solvent dielectric permittivity (Smith \& Deen 1980, 1983; Probstein 2003). The ion concentrations on both sides of the EGL are assumed to be the same, and the bulk concentration for species $i$ is expressed as $C_{\infty i}$. In the EGL, we assume equilibrium of ions, so that the Boltzmann distribution is realized:

$$
C_{i}=C_{\infty i} \exp \left[\frac{-F z_{i} \Psi}{R T}\right] .
$$

The neutrality condition requires

$$
\sum_{i} z_{i} C_{\infty i}=0
$$

In this study, for simplicity we consider a univalent-univalent electrolyte of the bulk ion concentration, $C_{\infty}$.

The electrostatic potential $\Psi$ around a solute, the surface charge densities on the cylinder, $q_{c}$, and the solute, $q_{s}$, and the electrostatic free energy $E$ are expressed in dimensionless form as $\Psi^{*}=F \Psi / R T, q_{c}^{*}=q_{c} L F / 2 \varepsilon R T, q_{s}^{*}=q_{s} L F / 2 \varepsilon R T$ and $E^{*}=2 E(F / R T)^{2} / \varepsilon L$, respectively. For a univalent-univalent electrolyte, the Debye length is defined as $\lambda_{D}=\left[\varepsilon R T / 2 F^{2} C_{\infty}\right]^{1 / 2}$. Note that $\lambda_{D} \approx 1 \mathrm{~nm}$ for a $0.1 \mathrm{M}$ aqueous solution at $T=310 \mathrm{~K}$. By using the Debye length, we introduce another dimensionless parameter $\tau=(L / 2) / \lambda_{D}$. In terms of these dimensionless quantities, we obtain a dimensionless form of the PB equation by combining (2.16) and (2.17):

$$
\nabla^{2} \Psi^{*}=\tau^{2} \sinh \Psi^{*}
$$

If $\left|\Psi^{*}\right| \ll 1$, then (2.19) can be approximated as

$$
\nabla^{2} \Psi^{*}=\tau^{2} \Psi^{*}
$$

This approximation is termed the DH approximation, and (2.20) is referred to as the $\mathrm{DH}$ equation, which we adopt in this study.

In general, the dielectric constants of macromolecules and fibres are much smaller than the dielectric constant of water. Thus, we assume that the dielectric constants of the cylinders and solutes are negligible compared to $\varepsilon$. In this case, the boundary conditions for (2.20) become

$$
\begin{array}{ll}
\partial_{n} \Psi^{*}=-q_{c}^{*} & \text { on the cylinder surface, } \\
\partial_{n} \Psi^{*}=-q_{s}^{*} & \text { on the solute surface, }
\end{array}
$$


where $\partial_{n}$ represents the derivative normal to the surface in the electrolyte region. Smith \& Deen (1980) showed that, for porous membranes, the use of either vanishing dielectric constants or a representative finite value $(=0.05 \times \varepsilon)$ for a solid material has little effect on the electrostatic potential in the water-filled pore, except for $\tau<2$.

By considering the charging process, Verwey \& Overbeek (1948) showed that the electrostatic free energy for a solid surface $\partial V$ at constant charge density $q^{*}$ may be calculated as

$$
E^{*}=\int_{\partial V} \mathrm{~d} S \int_{0}^{q^{*}} \Psi^{*} \mathrm{~d} q^{*} .
$$

Linearization of the $\mathrm{PB}$ equation ( $\mathrm{DH}$ approximation) ensures that the electrostatic potential is a linear function of the charge density. Therefore, (2.23) is reduced to

$$
E^{*}=\frac{q^{*}}{2} \int_{\partial V} \Psi^{*}\left(q^{*}\right) \mathrm{d} S .
$$

In this study, it is not straightforward to consider the charging process. However, Reiner \& Radke (1990) showed that, in the DH approximation, the electrostatic free energy obtained by the variational method coincides with (2.24) for a fixed charge density. Therefore, in this study, we adopt (2.24) to calculate the electrostatic free energy.

In terms of the electrostatic energy, $E^{*}$, the interaction energy or the excess free energy, between the solute and the cylinders can be expressed as follows:

$$
\phi^{*}=E_{C S}^{*}-E_{C}^{*}-E_{S}^{*},
$$

where subscript $C S$ designates the case in which both the cylinder and solute surfaces are electrically charged, subscript $C$ denotes the case in which only the charged cylinders are present and subscript $S$ denotes the case in which only the charged solute is present (Smith \& Deen 1980). For given positions of the solute centre $\left(c_{y}^{*}, c_{z}^{*}\right)$, we have computed the electrostatic potential and have estimated the electrostatic energy for the three cases $E_{C S}^{*}, E_{C}^{*}$ and $E_{S}^{*}$, so as to obtain the solute potential $\phi^{*}\left(c_{y}^{*}, c_{z}^{*}\right)$.

For the parameter values, we set $L=20 \mathrm{~nm}$ and $r_{f}=6 \mathrm{~nm}$ (i.e. $r_{f}^{*}=0.6$ ) (Squire et al. 2001), and $\varepsilon=6.57 \times 10^{-10} \mathrm{C}^{2}\left(\mathrm{~N} \cdot \mathrm{m}^{2}\right)^{-1}$, corresponding to aqueous solutions at $T=310 \mathrm{~K}$. At $L=20 \mathrm{~nm}$, the dimensionless charge density $q_{s}^{*}=q_{c}^{*}=1$ corresponds to $q_{s}=q_{c}=1.75 \times 10^{-3} \mathrm{C} \mathrm{m}^{-2}$. As discussed below, this value is comparable to charge densities estimated for the EGL and for albumin with $a=3.6 \mathrm{~nm}$ and a net charge of -17 . The ion concentrations $C_{\infty}=0.01,0.02,0.04,0.1$ and $0.15 \mathrm{M}$ correspond to $\tau=3.32,4.69,6.63,10.49$ and 12.84 , respectively.

\subsection{Numerical procedures}

Based on the formulation presented in $\S 2.3$, the value of $\sigma_{v}$ is obtained as a function of $a^{*}, q_{c}^{*}, q_{s}^{*}$ and $\tau$ by the following three steps.

(a) Evaluation of $\Psi^{*}$ using (2.20) through (2.22) for given $a^{*}, q_{c}^{*}$, $q_{s}^{*}$ and $\tau$ for various positions of the solute centre.

(b) Evaluation of $\phi^{*}$ using (2.24) and (2.25).

(c) Evaluation of $\sigma_{v}$ using (2.8), (2.10) and (2.15b).

The details in each step are shown elsewhere (Akinaga et al. 2008). Briefly, in step (a), (2.20) was solved subject to the boundary conditions (2.21) and (2.22) using a finite-element spectral method. We also applied the condition of $\partial \Psi^{*} / \partial x^{*}=0$ at the upstream and downstream cross-sections located at \pm 4 from the solute centre along the $x^{*}$-axis and adopted periodic conditions in the $y^{*}$ - and $z^{*}$-directions. 
The computational domain was divided into a number of elements having curved hexahedral shapes, within which the electrostatic potential was approximated by interpolation functions composed of Chebyshev polynomials of the eighth degree in the local coordinates. Special attention was given to the construction of elements near the boundaries of the cylinder and the solute surface, especially in the case of small Debye lengths. The numerical integrations in the finite-element scheme were performed by the Gauss-Legendre formulae of the 16th degree along each local coordinate axis. The numerical error was assessed by changing the truncation levels of the interpolation functions, similar to the method used by Akinaga et al. (2008).

In step $(b)$, we also adopted the Gauss-Legendre formulae of the 16th degree in the numerical integration that appeared in (2.24). In step (c), we solved (2.8) using a finite-element spectral scheme similar to that in step $(a)$, except for the fact that $(2.8)$ is two-dimensional.

In the computation of the electrostatic potential, the solute centre $\left(c_{y}^{*}, c_{z}^{*}\right)$ should cover the hatched region OAEF shown in figure $2(b)$. However, we have analysed only the cases in which the solute centre is located on lines OG and OA. Since line FG is equivalent to line $\mathrm{FE}$, and $\mathrm{e}^{-\phi^{*}} \rightarrow 0$ along the arc $\mathrm{AE}$ due to the steric condition, we obtained the values along the entire circumference of region OAEF. By using these values, we interpolated $\mathrm{e}^{-\phi^{*}}$ inside region $\mathrm{OAEF}$, as described in our previous paper (Sugihara-Seki 2006). When the solute centre is located on line OG, the position can be expressed as $\left(y^{*}, z^{*}\right)=\left(c_{y}^{*}, 0\right)$ with $0 \leqslant c_{y}^{*}<\sqrt{3}-r_{f}^{*}-a^{*}$, and on line OA as $\left(y^{*}, z^{*}\right)=\left(0, c_{z}^{*}\right)$, with $0 \leqslant c_{z}^{*}<1-r_{f}^{*}-a^{*}$. The relative error of $\exp \left[-\left(\phi^{*}\left(y^{*}, z^{*}\right)-\phi_{0}^{*}\right)\right]$ due to this interpolation was estimated to be at most a few per cent.

In this study, we limit our analysis to the case of $q_{c}^{*}, q_{s}^{*} \leqslant 0$. For the case of $q_{c}^{*}$, $q_{s}^{*} \geqslant 0$, the values of $E^{*}$ and $\phi^{*}$ are the same as those for the case in which $-q_{c}^{*},-q_{s}^{*}$, although the sign of $\Psi^{*}$ is changed. Accordingly, the results of $\sigma_{v}$ for $q_{c}^{*}$ and $q_{s}^{*}$ are the same as those for the case in which $-q_{c}^{*}$ and $-q_{s}^{*}$. We do not consider the case of $q_{c}^{*} \cdot q_{s}^{*}<0$, i.e. attractive interaction, because the assumption of a dilute solution used in this study may not be valid near the cylinder surface (Akinaga et al. 2008).

\section{Results}

As a representative example of our numerical results, a cross-sectional view of the electrostatic potential $\Psi^{*}$ is shown in figure 3, for the case in which the solute centre is placed at $\left(y^{*}, z^{*}\right)=(0.4,0)$ with $a^{*}=0.3, q_{c}^{*}=q_{s}^{*}=-5.7\left(q_{c}=q_{s}=-0.01 \mathrm{C} \mathrm{m}^{-2}\right)$ and $\tau=10.49$. Steep gradients of the electrostatic potential are observed near the cylinder and solute surfaces, and, apart from these boundaries, there are regions of gentle gradients of the potential. A decrease in $\tau\left(=L / 2 \lambda_{D}\right)$ can be shown to increase the region of steep gradients of $\Psi^{*}$ near the boundaries, which may be anticipated because the Debye length represents a characteristic distance of the charge effect from the surface charge or a characteristic double-layer thickness. Thus, the decrease in $\tau$ (increase in $\lambda_{D}$ ) implies an increase in electrostatic interaction between fixed surface charges.

Figure $4(a)$ shows the profiles of the Boltzmann factor, $\exp \left[-\left(\phi^{*}\left(y^{*}, z^{*}\right)-\phi_{0}^{*}\right)\right]$, along the $y^{*}$ - and $z^{*}$-axes for $a^{*}=0.3, q_{c}^{*}=q_{s}^{*}=-5.7$, and for various values of $\tau$. In the absence of the surface charge $\left(q_{c}^{*}=q_{s}^{*}=0\right)$, the steric condition leads to $\exp \left[-\left(\phi^{*}\left(y^{*}, z^{*}\right)-\phi_{0}\right)\right]=1$ for $0 \leqslant y^{*}<\sqrt{3}-a^{*}-r_{f}^{*} \approx 0.832$ along the $y^{*}$-axis or $0 \leqslant z^{*}<1-a^{*}-r_{f}^{*}=0.1$ along the $z^{*}$-axis, and $\exp \left[-\left(\phi^{*}\left(y^{*}, z^{*}\right)-\phi_{0}\right)\right]=0$ in the other ranges of $y^{*}$ and $z^{*}$. These profiles are plotted by thin dotted lines in figure $4(a)$. From the definition of $\phi_{0}^{*}, \exp \left[-\left(\phi^{*}\left(y^{*}, z^{*}\right)-\phi_{0}^{*}\right)\right]$ is unity at $\left(y^{*}, z^{*}\right)=(1 / \sqrt{3}, 0)$ or at point 


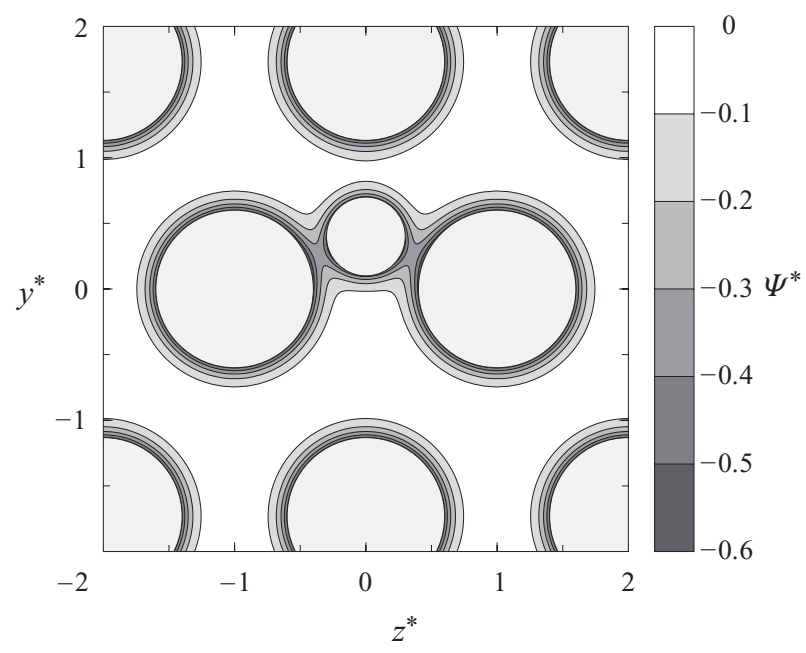

FIgURE 3. Cross-sectional view of the contours of the electrostatic potential $\Psi^{*}$ at a plane containing the solute centre located at $\left(y^{*}, z^{*}\right)=(0.4,0)$ with $a^{*}=0.3, q_{c}^{*}=q_{s}^{*}=-5.7$ and $\tau=10.49$.

$\mathrm{F}$ in figure 2. Figure 4(a) indicates that, at this point, the Boltzmann factor reaches its maximum or the solute potential, $\phi^{*}\left(y^{*}, z^{*}\right)$, reaches its minimum, which may be evident from the symmetry considerations. As shown in figure 4(a), the Boltzmann factor becomes minimum at the origin along the $y^{*}$-axis between the origin and point F. This minimum value, $\exp \left[-\left(\phi^{*}(0,0)-\phi_{0}^{*}\right)\right]$, increases with increasing $\tau$ up to 1 in the limit of $\tau$ approaching infinity.

In figure $4(b)$, the curves of $\exp \left(-\phi_{0}^{*}\right)$ are plotted for $a^{*}=0.1,0.2$ and 0.3 and $q_{c}^{*}=q_{s}^{*}=-5.7$. It is seen that $\exp \left(-\phi_{0}^{*}\right)$ decreases or $\phi_{0}^{*}$ increases, with decreasing $\tau$ and/or increasing $a^{*}$, which results from the enhancement of the electrostatic interaction between the solute and the cylinders.

Figure 5(a) shows the velocity profiles of $u_{2}^{*}$ along the $y^{*}$ - and $z^{*}$-axes for $a^{*}=0.3$, $q_{c}^{*}=q_{s}^{*}=-5.7$, and for various values of $\tau$. For comparison, the corresponding curves in the absence of surface charge $\left(q_{c}^{*}=q_{s}^{*}=0\right)$ are plotted by thin dotted lines. Further, the velocity profiles of $u_{1}^{*}$ are represented by thin solid lines, which are coincident with the solution given by Sparrow \& Loeffler Jr (1959). As expected, the velocity profiles $u_{1}^{*}$ and $u_{2}^{*}$ achieve the maximum at $\left(y^{*}, z^{*}\right)=(1 / \sqrt{3}, 0)$ (point $\mathrm{F}$ ). Equation $(2.8 b)$ indicates that the Boltzmann factor, $\exp \left[-\left(\phi^{*}\left(y^{*}, z^{*}\right)-\phi_{0}^{*}\right)\right]$, drives the flow of $u_{2}^{*}$. As shown in figure $4(a)$, a decrease in $\tau$ diminishes the driving force for $u_{2}^{*}$, which results in a decrease in $u_{2}^{*}$. This trend implies an increase in the osmotic flow, because the osmotic flow rate is proportional to $U_{1}^{*}-U_{2}^{*} \exp \left(-\phi_{0}^{*}\right)$, where $U_{1}^{*}$ and $U_{2}^{*}$ are the flow rates of $u_{1}^{*}$ and $u_{2}^{*}$, respectively (see (2.13)). In other words, an increase in the electrostatic interaction with decreasing $\tau$ enhances the osmotic flow for fixed surface charges.

In order to better understand the velocity field, the contours of $u_{2}^{*}$ at a plane perpendicular to the $x^{*}$-axis are plotted in figure $5(b)$ for $a^{*}=0.3, q_{c}^{*}=q_{s}^{*}=-5.7$ and $\tau=10.39$. The velocity profiles along the $y^{*}$ - and $z^{*}$-axes in figure $5(b)$ correspond to the dotted curves in figure $5(a)$. Figure $5(b)$ indicates that the velocity contours around each cylinder are nearly circular, except in the region near point $F$ (cross symbols in figure $5 b$ ). 

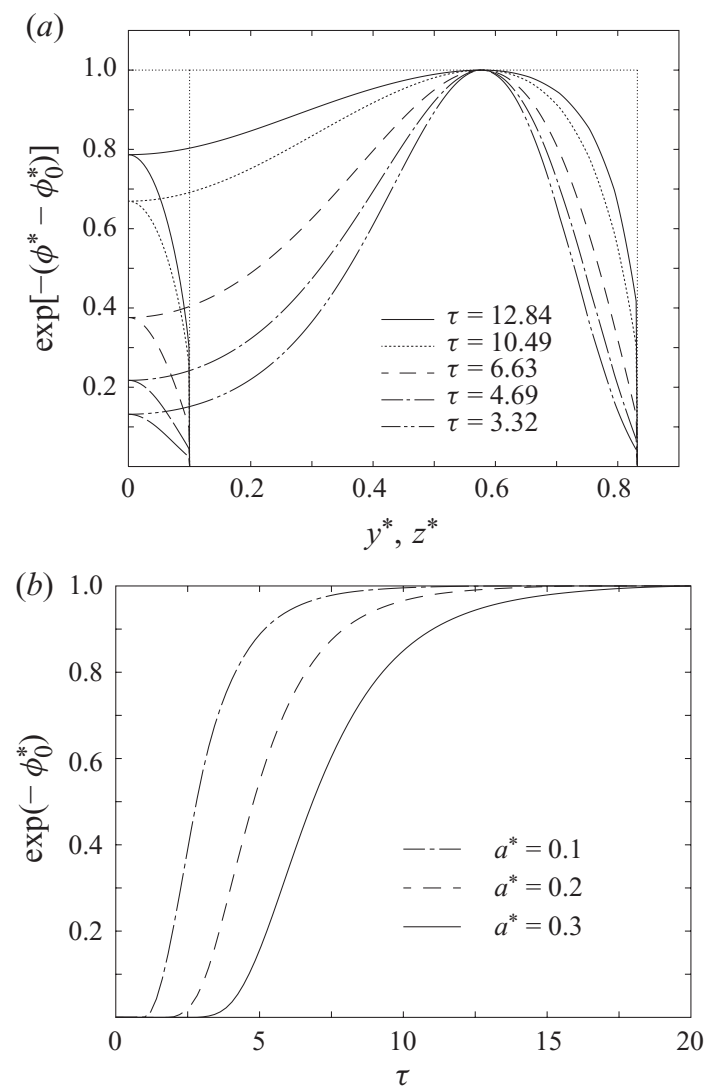

Figure 4. (a) Profiles of the Boltzmann factor, $\exp \left[-\left(\phi^{*}\left(y^{*}, z^{*}\right)-\phi_{0}^{*}\right)\right]$, along the $y^{*}$ - and $z^{*}$-axes for $a^{*}=0.3, q_{c}^{*}=q_{s}^{*}=-5.7$ and $\tau=3.32,4.69,6.63,10.49$ and 12.84. The thin dotted lines represent the corresponding lines in the absence of the surface charge $\left(q_{c}^{*}=q_{s}^{*}=0\right)$. (b) $\exp \left(-\phi_{0}^{*}\right)$ as a function of $\tau$ at $q_{c}^{*}=q_{s}^{*}=-5.7$, where $\phi_{0}^{*}$ represents the solute potential at point $\mathrm{F}$ (see figure 2 ).

The obtained values of the reflection coefficient $\sigma_{v}$ are plotted in figure 6 as a function of the solute size, $a^{*}$, for $q_{s}^{*}=-5.7$ and for three different values of $q_{c}^{*}$ $(=-0.51,-5.7$ and -15.2$)$ for various values of $\tau$. The values of $q_{c}^{*}$ are chosen for later discussion in $\S 4$. For reference, the reflection coefficient in the absence of the surface charge $\left(q_{c}^{*}=q_{s}^{*}=0\right)$ is plotted by a thin dotted line. The corresponding curve, which was expressed in a simple analytical form by Zhang et al. (2006), is shown by a thin solid line. These two curves agree closely with each other. Figure 6 shows that the curve given by the thin dotted line is lower than the other curves, indicating that the repulsive electrostatic interaction between the solute and cylinder surface charges increases the reflection coefficient $\sigma_{v}$. Figure 6 indicates a monotonic increase in $\sigma_{v}$ with decreasing $\tau$ or increasing Debye length for constant $a^{*}$. This tendency is intuitive because an increase in Debye length is qualitatively similar to an increase in the solute radius. Analogously, an elevation in the surface charge density strengthens the electrostatic interaction, thereby augmenting $\sigma_{v}$.

Figure $7(a-c)$ illustrates the dependence of $\sigma_{v}$ on the surface charge densities, $q_{s}^{*}$ and $q_{c}^{*}$, for $a^{*}=0.1,0.2$ and 0.3 at $\tau=3.32$ and 10.49. These figures indicate that the electrostatic effect becomes more significant for larger surface charges, as mentioned 

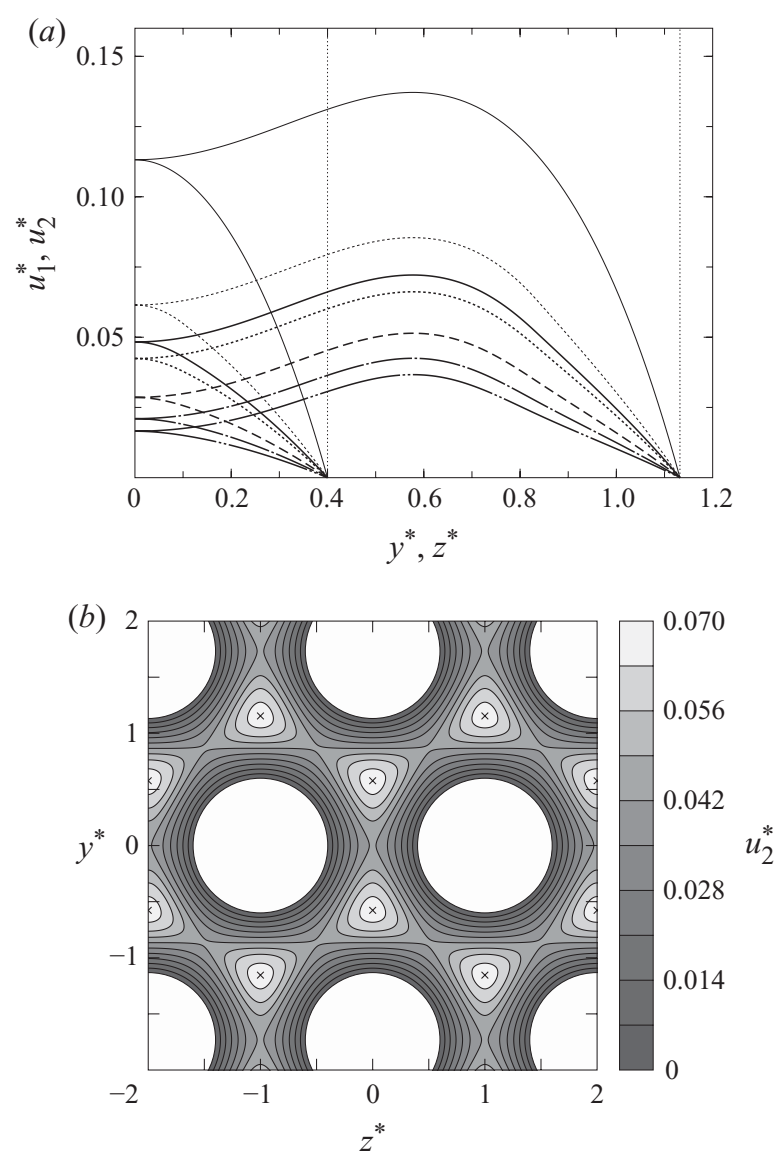

FIGURE 5. (a) Velocity profiles of $u_{2}^{*}$ along the $y^{*}$ - and $z^{*}$-axes for $a^{*}=0.3, q_{c}^{*}=q_{s}^{*}=-5.7$ and $\tau=3.32$ (dash-double-dotted lines), 4.69 (dash-dotted lines), 6.63 (dashed lines), 10.49 (dotted lines) and 12.84 (solid lines). The corresponding curves in the absence of the surface charge $\left(q_{c}^{*}=q_{s}^{*}=0\right)$ are plotted by thin dotted lines. The velocity profiles of $u_{1}^{*}$ are plotted by thin solid lines. (b) Contours of velocity $u_{2}^{*}$ at a plane perpendicular to the $x^{*}$-axis for $a^{*}=0.3$, $q_{c}^{*}=q_{s}^{*}=-5.7$ and $\tau=10.49$. The points corresponding to point $\mathrm{F}$ are indicated by cross symbols.

above. In addition, it is found that, at $\tau=10.49$, the $\sigma_{v}$ values increase approximately linearly with increasing $\left|q_{c}^{*}\right|$ in the range examined, whereas they steeply increase and then gradually approach 1 at $\tau=3.32$. These trends are also observed when $\left|q_{s}^{*}\right|$, instead of $\left|q_{c}^{*}\right|$, is increased at constant $q_{c}^{*}$ (not shown). Comparison of the variations of $\sigma_{v}$ with respect to $q_{s}^{*}$ (at constant $q_{c}^{*}$ ) and with respect to $q_{c}^{*}$ (at constant $q_{s}^{*}$ ) reveals that the influences of $q_{s}^{*}$ and $q_{c}^{*}$ on $\sigma_{v}$ are comparable.

\section{Discussion}

An electrostatic model was developed to predict the effects of surface charge on the osmotic reflection coefficient of charged spherical solute across the EGL, based on the combination of low-Reynolds-number hydrodynamics and a continuum description of the electric double layers. 


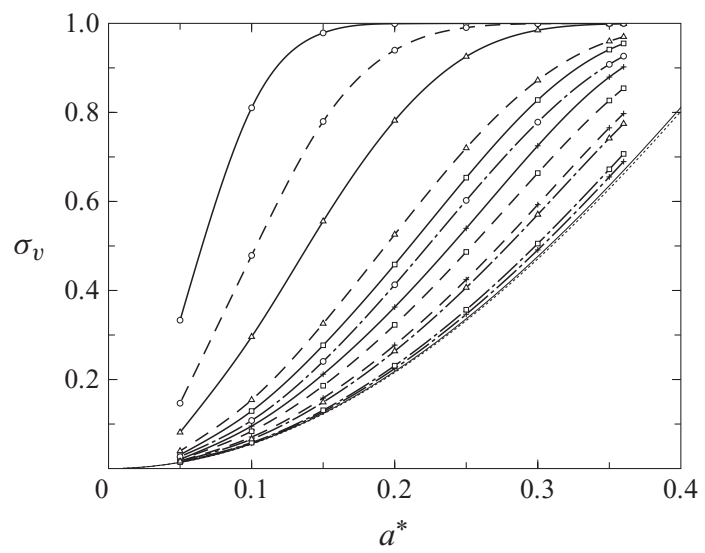

FIGURE 6. Reflection coefficient $\sigma_{v}$ at $q_{s}^{*}=-5.7$ and $q_{c}^{*}=-0.51$ (dash-dotted lines), -5.7 (dashed lines) and -15.2 (solid lines) for $\tau=3.32$ (open circle symbols), 6.63 (triangle symbols), 10.49 (square symbols) and 12.84 (plus symbols). For reference, $\sigma_{v}$ for the steric condition alone is plotted by a thin dotted line. The corresponding curve given in Zhang et al. (2006, equation 28) is plotted by a thin solid line.

We have tried to determine the parameter values, $q_{c}^{*}$ and $q_{s}^{*}$, under physiological conditions. Using a Donnan-type model for electrostatic partitioning, the charge density in the microvessel wall of the frog mesentery was estimated by Adamson et al. (1988) to be approximately $-11.4 \mathrm{mEq}^{-1}$ and by Curry et al. (1989) to be in the range from -11 to $-28 \mathrm{mEq}^{-1}$. Based on the experimental data for frog mesenteric capillaries and their electrodiffusion model for the EGL, Fu et al. (2003) estimated the charge density of EGL to be -25 to $-35 \mathrm{mEq}^{-1}$. By comparing the EGL recovery time obtained by their mechano-electrochemical model of the EGL with experimental observation, Damiano \& Stace (2002) estimated the charge density of the EGL to be approximately $-1 \mathrm{mEq}^{-1}$. If we assume in the present model that all of the electric charge in the EGL is distributed uniformly on the surface of the cylinders, then the surface charge density, $q_{c}$, on the cylinder surface can be expressed in terms of the volumetric charge density, $\rho$, as $q_{c}=\sqrt{3} \rho L^{2} /\left(4 \pi r_{f}\right)$. This assumption leads to the following: the charge densities of $\rho=-1,-11.3$ and $-30 \mathrm{mEq}^{-1}$ in the EGL correspond to $q_{c}=-0.89 \times 10^{-3} \mathrm{C} \mathrm{m}^{-2}\left(q_{c}^{*}=-0.51\right),-1.00 \times 10^{-2} \mathrm{C} \mathrm{m}^{-2}\left(q_{c}^{*}=-5.7\right)$ and $-2.66 \times 10^{-2} \mathrm{C} \mathrm{m}^{-2}\left(q_{c}^{*}=-15.2\right)$, respectively, at $L=20 \mathrm{~nm}$ and $r_{f}=6 \mathrm{~nm}$. In figure 6 , the $\sigma_{v}$ values obtained for these $q_{c}^{*}$ are plotted by dash-dotted lines, dashed lines and solid lines, respectively.

With regard to the charge density on the solute $q_{s}$, we assumed that all of the electric charge in the solute is distributed uniformly over the spherical surface. For example, $q_{s}=-1.7 \times 10^{-2} \mathrm{C} \mathrm{m}^{-2}\left(q_{s}^{*}=-9.5\right)$ for albumin with $a=3.6 \mathrm{~nm}$ and net charge $=-17$ and $q_{s}=-3.4 \times 10^{-2} \mathrm{C} \mathrm{m}^{-2}\left(q_{s}^{*}=-19.6\right)$ for $\alpha$-lactalbumin with $a=2.0 \mathrm{~nm}$ and net charge $=-11$.

By using these charge densities, we compare the values obtained for $\sigma_{v}$ at $\tau=12.84$ $\left(C_{\infty}=0.15 \mathrm{M}\right)$ with the experimental measurements. The experimental value of $\sigma_{v}$ for albumin (Stokes radius $=3.6 \mathrm{~nm}$ ) is typically $>0.9$ (Michel \& Curry 1999). Figure 6 shows that $\sigma_{v}=0.662$ for $a^{*}=0.36(a=3.6 \mathrm{~nm})$ without charge effects $\left(q_{c}^{*}=q_{s}^{*}=0\right)$, which is far smaller than the measured values. In the charged case of $q_{s}^{*}=-5.7$ at $a^{*}=0.36$, we obtain $\sigma_{v}=0.690,0.797$ and 0.902 for $q_{c}^{*}=-0.51\left(\rho=-1 \mathrm{mEq} 1^{-1}\right)$, $-5.7\left(\rho=-11.3 \mathrm{mEq}^{-1}\right)$ and $-15.2\left(\rho=-30 \mathrm{mEq} \mathrm{l}^{-1}\right)$, respectively. In order to 

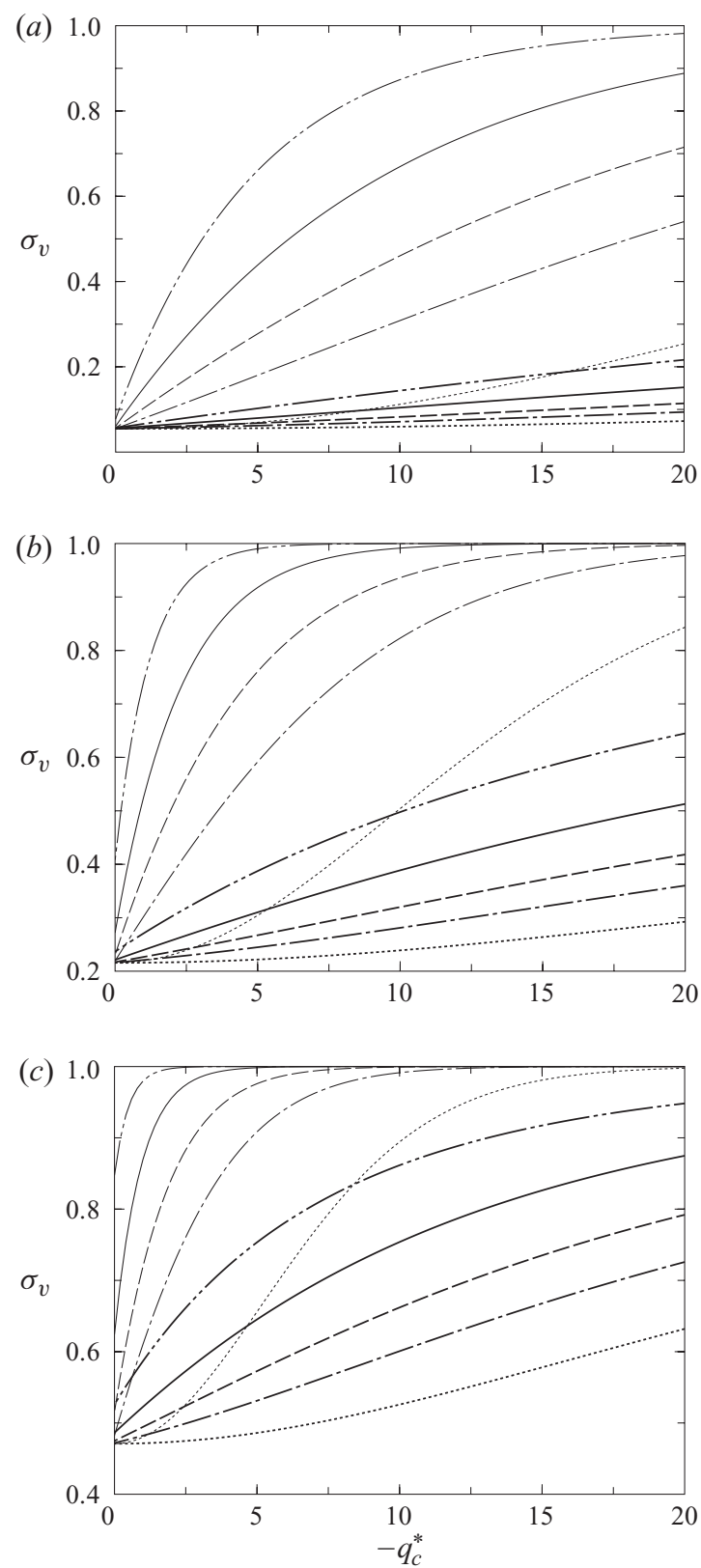

FIGURE 7. Reflection coefficient $\sigma_{v}$ as a function of $-q_{c}^{*}$ for various values of $q_{s}^{*}$ at $(a) a^{*}=0.1$, (b) $a^{*}=0.2$ and $(c) a^{*}=0.3$. In each part, the dotted lines represent the case of $q_{s}^{*}=0$, the dash-dotted lines represent the case of $q_{s}^{*}=-1.4$, the dashed lines represent the case of $q_{s}^{*}=-2.9$, the solid lines represent the case of $q_{s}^{*}=-5.7$ and the dash-double-dotted lines represent the case of $q_{s}^{*}=-11.4$. The thick lines denote the case of $\tau=10.49$ and the thin lines denote the case of $\tau=3.32$.

achieve a more precise comparison, we computed the case in which $q_{s}^{*}=-9.5$ with the other parameters unchanged, and the results are summarized in table 1. Although $\sigma_{v}$ for $q_{c}^{*}=-0.51$ appears low compared to the experimental measurements, the other two cases, in which $q_{c}^{*}=-5.7$ and -15.2 , provide a better prediction of 


\begin{tabular}{ccccc} 
& $a^{*}=0.36$ & \multicolumn{3}{c}{$a^{*}=0.2$} \\
\cline { 3 - 5 }$q_{c}^{*} q_{s}^{*}$ & -9.5 & 0 & -5.7 & -19.6 \\
0 & 0.699 & 0.216 & 0.219 & 0.253 \\
-0.51 & 0.718 & 0.216 & 0.225 & 0.268 \\
-5.7 & 0.849 & 0.221 & 0.278 & 0.394 \\
-15.2 & 0.942 & 0.247 & 0.364 & 0.540 \\
Experiments & $>0.9^{a}$ & $0.21^{b}$ & $0.29^{\dagger b}$ & $0.35^{c}$ \\
& & & & $0.34-0.69^{d}$
\end{tabular}

${ }^{a}$ Serum albumin (Michel \& Curry 1999), ${ }^{b}$ myoglobin (Michel \& Turner 1981), ${ }^{c} \alpha$-lactalbumin (Huxley et al. 1987), ${ }^{d} \alpha$-lactalbumin (Huxley et al. 1993). $\uparrow q_{s}^{*}$ : unknown.

TABLE 1. Reflection coefficient $\sigma_{v}$ at $\tau=12.84$.

$\sigma_{v}$ for albumin. Another comparison with the experimental measurements may be possible for myoglobin (Stokes radius $=1.9 \mathrm{~nm}$ ). The osmotic reflection coefficient, $\sigma_{v}$, measured for anionized myoglobin has been reported to be 0.29 , whereas $\sigma_{v}$ for neutral myoglobin has been reported to be 0.21 (Michel \& Turner 1981). As shown in table 1 , the predicted values of $\sigma_{v}$ for the uncharged solute $\left(q_{s}^{*}=0\right)$ at $a^{*}=0.2$ are close to 0.21 , regardless of $q_{c}^{*}$. For charged solute with $q_{s}^{*}=-5.7$, the values of $\sigma_{v}$ are found to increase sufficiently to account for the experimental observation for the cases in which $q_{c}^{*}=-5.7$ and -15.2 , although the charge density of anionized myoglobin is not known. The following comparison is for $\alpha$-lactalbumin (Stokes radius $=2.0 \mathrm{~nm}$ ). As shown in table 1 , the values of $\sigma_{v}$ for $q_{c}^{*}=-5.7$ and -15.2 are comparable to the measured $\sigma_{v}$ in frog mesenteric capillaries, which is 0.35 (Huxley, Curry \& Adamson 1987) and $0.34 \simeq 0.69$ (Huxley et al. 1993). In all of the cases considered, the present analyses performed by taking into account the electrostatic interaction provide a much better description of $\sigma_{v}$, as compared to the analyses performed without considering charge effects. In particular, in the cases of $q_{c}^{*}=-5.7$ or -15.2 , i.e. the charge density $\rho \approx-10$ or $-30 \mathrm{mEq}^{-1}$ in the EGL, the predicted values of $\sigma_{v}$ appear to show good agreement with the experimental measurements.

This study is based on a continuum description of the electric double layer, in which ions were assumed to be sufficiently small that the electrolyte can be regarded as a continuum and the electric field was determined on the basis of the mean-field theory (Verwey \& Overbeek 1948; Probstein 2003). However, the ions are of finite size, and this deficiency may be corrected, to a certain extent, by considering explicitly the formation of Stern layers near the surfaces (Probstein 2003). As noted in $\S 1$, recent studies on charged biomaterials have reported anomalous behaviours caused by charge correlation effects, which the mean-field theory fails to describe. Although we cannot deny the possibility that charge correlations may play a role in the solute behaviour in the EGL, we have presented the current model as the simplest and most fundamental model to explore charge effects on the osmotic flow across the EGL.

In general, the osmotic flow across a membrane with charged pores creates convection of counterions, which generates an electric current along the pore. If the zero-current condition is imposed, a potential gradient (streaming potential) must develop such that the total current due to ion migration balances that due to 
convection. Quite recently, Bhalla \& Deen (2009) investigated a similar problem to that investigated by Akinaga et al. (2008), including the streaming potential, and showed that the streaming potential has little effect on the osmotic reflection coefficients, $\sigma_{v}$, for membranes with circular cylindrical pores. We found that the values they obtained for $\sigma_{v}$ exhibited excellent agreement with the corresponding values of Akinaga et al. (2008). Thus, we would not expect the present results to be changed markedly if they are applied to the cases in which the streaming potential should be included. Bhalla $\&$ Deen (2009) also examined the difference between the solutions of the PB equation and the $\mathrm{DH}$ equation, and concluded that the $\mathrm{DH}$ approximation provides $\sigma_{v}$ with sufficient accuracy, even in the cases of rather high surface charge densities and/or large Debye lengths. We also confirmed this conclusion for the same geometry, by comparing the results obtained from the PB equation in Akinaga et al. (2008) and those obtained from the DH equation. Therefore, in this study, we adopted the DH approximation. Under this approximation, the analysis is much easier compared to that for the PB equation, and the electrostatic free energy is well defined. It remains for future studies to verify the validity of applying the $\mathrm{DH}$ equation to the present problem, especially in the case of high surface charge densities.

The endothelial surface glycocalyx is composed of a wide variety of membranebound macromolecules, including glycoproteins bearing acidic oligosaccharides with terminal sialic acids and proteoglycans with their associated glycosasminoglycan side chains. The polyanionic nature of these constituents imparts a net negative charge. These macromolecules form a network in which soluble molecules, either plasma- or endothelium-derived, are incorporated. This layer is further extended in plasma by association with proteins and other plasma components to form an EGL. Excellent reviews of the compositions and functions of the EGL were recently provided (Pries et al. 2000; Weinbaum et al. 2003, 2007; Reitsma et al. 2007).

Through a detailed structural analysis using computed autocorrelation functions and Fourier transforms of electron micrograph images, Squire et al. (2001) indicated the quasi-periodic structure of the EGL. The EGL is a three-dimensional fibrous network with focal scattering centres of $10-12 \mathrm{~nm}$ in diameter with a characteristic spacing of $20 \mathrm{~nm}$ in both parallel and perpendicular directions to the endothelial surface. Based on these findings, they proposed an ultrastructural model for the EGL, where clusters of fibrous strands of $10-12 \mathrm{~nm}$ in diameter are projected normally to the endothelial surface to form a regular lattice with a spacing of $20 \mathrm{~nm}$. This basic ultrastructural model was simplified to the idealized mathematical model by replacing the core protein strands with circular cylinders arranged in an ordered hexagonal fashion (see figure 1), and this idealized model was used to examine various properties of the EGL, including its permeability (Weinbaum et al. 2003; Sugihara-Seki 2006; Zhang et al. 2006).

In this study, we adopted this idealized model as an initial attempt to examine the charge effect on the osmotic flow across the EGL, by assuming that the electric charge is distributed uniformly on the surfaces of the cylinder and the solute. The charge distributions were assumed to be fixed, so that their distortions or rearrangements possibly induced by the electrostatic interaction were not included. We also neglected detailed structures in the EGL, its flexible property, as well as the effects of adsorbed plasma proteins (Vink \& Duling 1996, 2000; Pries et al. 2000; Stace \& Damiano 2001; Damiano \& Stace 2002; Weinbaum et al. 2003, 2007; Reitsma et al. 2007). Additional studies considering these effects are necessary in order to arrive at a more comprehensive understanding of the charge effect on the osmotic flow across the EGL. 


\section{Conclusions}

We have developed an electrostatic model for osmotic flow across a layer consisting of hexagonally ordered circular cylinders, and the results are applied to the osmotic reflection coefficients of charged macromolecules across the EGL. Comparison with previous estimates in the absence of electric effects reveals that our model provides an improved description of the reflection coefficients and accounts well for the experimentally obtained values for charged macromolecules, such as serum albumin.

This research has been supported in part by the Grant-in-Aid for Scientific Research (B) (No. 19360090) from JSPS and the Special Research Fund, Kansai University.

\section{REFERENCES}

Adamson, R. H., Huxley, V. H. \& Curry, F. E. 1988 Single capillary permeability to proteins having similar size but different charge. Am. J. Physiol. 254, H304-H312.

Akinaga, T., Sugihara-Seki, M. \& Itano, T. 2008 Electrical charge effect on osmotic flow through pores. J. Phys. Soc. Japan 77, 053401.

Anderson, J. L. \& Malone, D. M. 1974 Mechanism of osmotic flow in porous membranes. Biophys. J. 14, 957-982.

Bhalla, G. \& DeEn, W. M. 2009 Effects of charge on osmotic reflection coefficients of macromolecules in porous membranes. J. Colloid Interface Sci. 333, 363-372.

CURRY, F. E. 1984 Mechanics and thermodynamics of transcapillary exchange. In Handbook of Physiology, Section 2: The Cardiovascular System (ed. E. M. Renkin \& C. C. Michel), vol. 4, pp. 309-374. American Physiological Society.

Curry, F. E., Rutledge, J. C. \& Lenz, J. F. 1989 Modulation of microvessel wall charge by plasma glycoprotein orosomucoid. Am. J. Physiol. 257, H1354-H1359.

Damiano, E. R. \& Stace, T. M. 2002 A mechano-electrochemical model of radial deformation of the capillary glycocalyx. Biophys. J. 82, 1153-1175.

Fu, B. M., Chen, B. \& Chen, W. 2003 An electrodiffusion model for effects of surface glycocalyx layer on microvessel permeability. Am. J. Physiol. 284, H1240-H1250.

Huxley, V. H. \& CuRry, F. E. 1991 Differential actions of albumin and plasma on capillary solute permeability. Am. J. Physiol. 260, H1645-H1654.

Huxley, V. H., Curry, F. E. \& Adamson, R. H. 1987 Quantitative fluorescence microscopy on single capillaries: $\alpha$-lactalbumin transport. Am. J. Physiol. 252, H188-H197.

Huxley, V. H., Curry, F. E., Powers, M. R. \& Thipakorn, B. 1993 Differential action of plasma and albumin on transcapillary exchange of anionic solute. Am. J. Physiol. 264, H1428-H1437.

Kedem, O. \& Katchalsky, A. 1958 Thermodynamic analysis of the permeability of biological membranes to non-electrolytes. Biochim. Biophys. Acta 27, 229-246.

Levin, Y. 2002 Electrostatic correlations: from plasma to biology. Rep. Prog. Phys. 65, 1577-1632.

LevitT, D. G. 1975 General continuum analysis of transport through pores. Part 1. Proof of Onsager's reciprocity postulate for uniform pore. Biophys. J. 15, 533-551.

Likos, C. N. 2001 Effective interactions in soft condensed matter physics. Phys. Rep. 348, 267439.

Michel, C. C. \& Curry, F. E. 1999 Microvascular permeability. Physiol. Rev. 79, 703-761.

Michel, C. C. \& Turner, M. R. 1981 The effects of molecular charge on the permeability of frog mesenteric capillaries to myoglobin. J. Phyisol. 316, 51P-52P.

Pries, A. R., Secomb, T. W. \& Gaehtgens, P. 2000 The endothelial surface layer. Pflügers Archiv: Eur. J. Physiol. 440, 653-666.

Probstein, R. F. 2003 Physicochemical Hydrodynamics. Wiley.

REINER, E. S. \& RADKE, C. J. 1990 Variational approach to the electrostatic free energy in charged colloidal suspensions: general theory for open systems. J. Chem. Soc. Faraday Trans. 86, 3901-3912.

ReINER, E. S. \& RADKe, C. J. 1991 Electrostatic interactions in colloidal suspensions: tests of pairwise additivity. AIChE J. 37, 805-824. 
Reitsma, S., Slaaf, D. W., Vink, H., van Andvoort, M. A. M. J. \& oude Egbrink, M. G. A. 2007 The endothelial glycocalyx: composition, functions, and visualization. Pflügers Archiv: Eur. J. Physiol. 454, 345-359.

Smith, F. G. III \& DeEN, W. M. 1980 Electrostatic double-layer interactions for spherical colloids in cylindrical pores. J. Colloid Interface Sci. 78, 444-465.

Smith, F. G. III \& DeEn, W. M. 1983 Electrostatic effects on the partitioning of spherical colloids between dilute bulk solution and cylindrical pores. J. Colloid Interface Sci. 91, 571-590.

Sparrow, E. M. \& LoefFler JR, A. L. 1959 Longitudinal laminar flow between cylinders arranged in regular array. AIChE J. 5, 325-330.

Squire, J. M., Chew, M., Nneji, G., Neal, C., Barry, J. \& Michel, C. 2001 Quasi-periodic substructure in the microvessel endothelial glycocalyx: a possible explanation for molecular filtering? J. Struct. Biol. 136, 239-255.

Stace, T. M. \& Damiano, E. R. 2001 An electrochemical model of the transport of charged molecules through the capillary glycocalyx. Biophys. J. 80, 1670-1690.

Sugihara-SeKi, M. 2006 Transport of spheres suspended in the fluid flowing between hexagonally arranged cylinders. J. Fluid Mech. 551, 309-321.

Sugihara-Seki, M., Akinaga, T. \& Itano, T. 2008 Flow across microvessel walls through the endothelial surface glycocalyx and the interendothelial cleft. J. Fluid Mech. 601, 229-252.

Truskey, G. A., Yuan, F. \& Katz, D. F. 2004 Transport Phenomena in Biological Systems. Pearson Education.

Ueda, A., Shimomura, M., Ikeda, M., Tanishita, R. \& Yamaguchi, K. 2004 Effect of glycocalyx on shear-dependent albumin uptake in endothelial cells. Am. J. Physiol. 287, H2287-H2294.

Verwey, E. J. W. \& Overbeek, J. T. G. 1948 Theory of the Stability of Lyophobic Colloids. Dover.

VINK, H. \& Duling, B. R. 1996 Identification of distinct luminal domains for macromolecules, erythrocytes, and leukocytes within mammalian capillaries. Circ. Res. 79, 581-589.

VINK, H. \& Duling, B. R. 2000 Capillary endothelial surface layer selectively reduces plasma solute distribution volume. Am. J. Physiol. 278, H285-H289.

Weinbaum, S., Tarbell, J. M. \& Damiano, E. R. 2007 The structure and function of the endothelial glycocalyx layer. Annu. Rev. Biomed. Engng 9, 121-167.

Weinbaum, S., Zhang, X., Han, Y., Vink, H. \& Cowin, S. C. 2003 Mechanotransduction and flow across the endothelial glycocalyx. Proc. Natl Acad. Sci. 100, 7988-7995.

Zhang, X., Curry, F. \& Weinbaum, S. 2006 Mechanism of osmotic flow in a periodic fibre array. Am. J. Physiol. 290, H844-H852. 